Ann. Abeille, 1963, 6, (4), 249-255.

\title{
SUR L'ORIGINE DES MACLES D'OXALATE DE CAI CIUM CONTENUES DANS LES MIELS DE TILLEUL
}

\author{
Z. DEMIANOWIC $\%$ \\ Centre apicole, Institul d'Arboriculture fruitière, Skierniencice (I'ologne)
}

SOMMAIRF

L'auteur ayant mis en évidence, en 1962 , la rrésence de macles d'oxalate de calcium dans les miels de 'Tilleul, a recherché leur origine et explicque par quel processus elles parviennent dans le miel.

\section{INTRODTUTION}

MaURizıo (I956) a signalé que, dans le pollen des plantes du gente Impatiens, aussi bien d'ailleurs chez les espèces sauvages que chez les Balsamines cultivées, on trouve des cellules contenant des raphides d'oxalate de calcium.

Peirimon (I960) de son côté a décrit dans des miels provenant de 'Tulcea (Roumanie) des cristaux en forme de rosettes. Il s'agit vraisemblablement de ces macles d'oxala'e de calcium dont j'ai montré (Demiaxowrcz, 1962) la présence dans tous les miels de Tilleul que j'ai analysés.

KÜSTER F. (I956) a consacré dans son livre "La cellule végétale " un chapitre assez important aux cri staux d'oxalate de calcium. Il commente la présence de ces cristaux aussi bien chez les Cryptogames que chez de nombreuses Phanérogames. Il décrit abondamment l'aspect des cristaux dont la présence est en rapport avec la teneur en eau, précise les conditions dans lesquelles ils apparaissent et leur position dans la cellule. Il cite abondamment les différents auteurs qui ont étudié l'apparition de cristaux d'oxalate de calcium au cours des différents stades du développement, leur résorption dans le stade final et aussi la signification écologique de leur apparition. Il décrit, par exemple le rôle protecteur des raphides contre les escargots, 
le rôle des gros cristaux simples comme réflecteurs de la lumière à l'intérieur du tissu et également leur fonction comme éléments de soutien mécanique dans les fibres.

Gorskr F. (I959) dans son traité de Physiologie végétale, fournit au chapitre consacré à l'excrétion interne des ions calcium et silicium trois hypothèses susceptibles d'expliquer l'apparition des cristaux d'oxalate de calcium. Selon la première de ces hypothèses, la fonction d'excrétion interne aurait pour effet d'immobiliser la substance nocive que constitue l'acide oxalique. Selon la seconde, elle permet d'éliminer de la plante des ions calcium qui se trouvent en excès. Les partisans de la troisième hypothèse considèrent la formation d'oxalate de calcium comme la conséquence inévitable de la présence concomitante dans la cellule d'ions calcium et d'acide oxalique.

\section{MATÉRIEL FT MÉTHODE}

J'ai utilisé les bourgeons floraux conservés dans l'alcool de trois des espéces de Tilleuls les plus fréquents en Pologne, à savoir : Tilia cordata, $T$. cuchlora, T. platyphyllos. Ces bourgeons avaient été récoltés en 1954 et en 1962 à différents stades de la floraison en trois endroits différents (près de lublin, à Skierniewice et à Krynica Morska). l)'autre part, j’ai exaniné le pollen de 12 espèces de T'illeul provenant d'herbiers, et le pollen frais de Sparmania africana (f. Tiliaceac), ainsi que celui de plantes mellifères importantes telles que le Trèfle, le Colzal, le sarrasin, etc.

A partir des bourgcons conservés dans l'alcool, j'ai confectionné des préparat ions histologirues selon la technique habituelle d'inclusion dans la paraffine et avec une coloration au vert malachite et au violet de gentiane. Les préparations obtenues ont été examinées en lumière normale et en lumière polarisée, ainsi d'ailleurs que les préparations de pollen frais.

\section{RÉSULTATS}

La paroi de l'anthère du Tilleul se compose de quatre couches, comme chez la plupart des plantes. Iintre les sacs polliniques se trouve un parenchyme. Dans beaucoup de cellules et dès les premiers stades du développement, se forment des macles d'oxalate de calcium (fig. I et 2). Souvent, elles apparaissent aussi dans la paroi médiane et dans le connectif. Après la maturation des anthères et le développement complet de la couche fibreuse, le parenchyme bourré de macles se trouve déchiré. Il en résulte qu'entre les sacs polliniques et la paroi externe encore intacte de l'anthère, il se forme un vide dans lequel pénètrent les macles (fig. 3 et 4 ). A l'ouverture de l'anthère, celles-ci sont expulsées vers l'extérieur avec les grains de pollen et peuvent venir saupoudrer le nectar ou bien se trouver récolté s avec le pollen par les abeilles et pénétrer ainsi dans les pelotes de pollen, puis dans les cellules.

L'examen anatomique des anthères de trois espèces de Tilleuls provenant de localités ayant des sols différents, récoltés dans des années différentes et par des temps différents, a montré l'identité de structure et la constance de la présence des macles d'oxalate de calcium dans le tissu qui se trouve entre les sacs polliniques.

Je dois souligner que les macles sont très faciles à reconnaître et à compter lorsqu'on examine la préparation sous le microscope en lumière polarisée. L'examen du pollen des I 2 espèces de Tilleul récolté en herbier a confirmé le caractère très général de la présence des macles. 


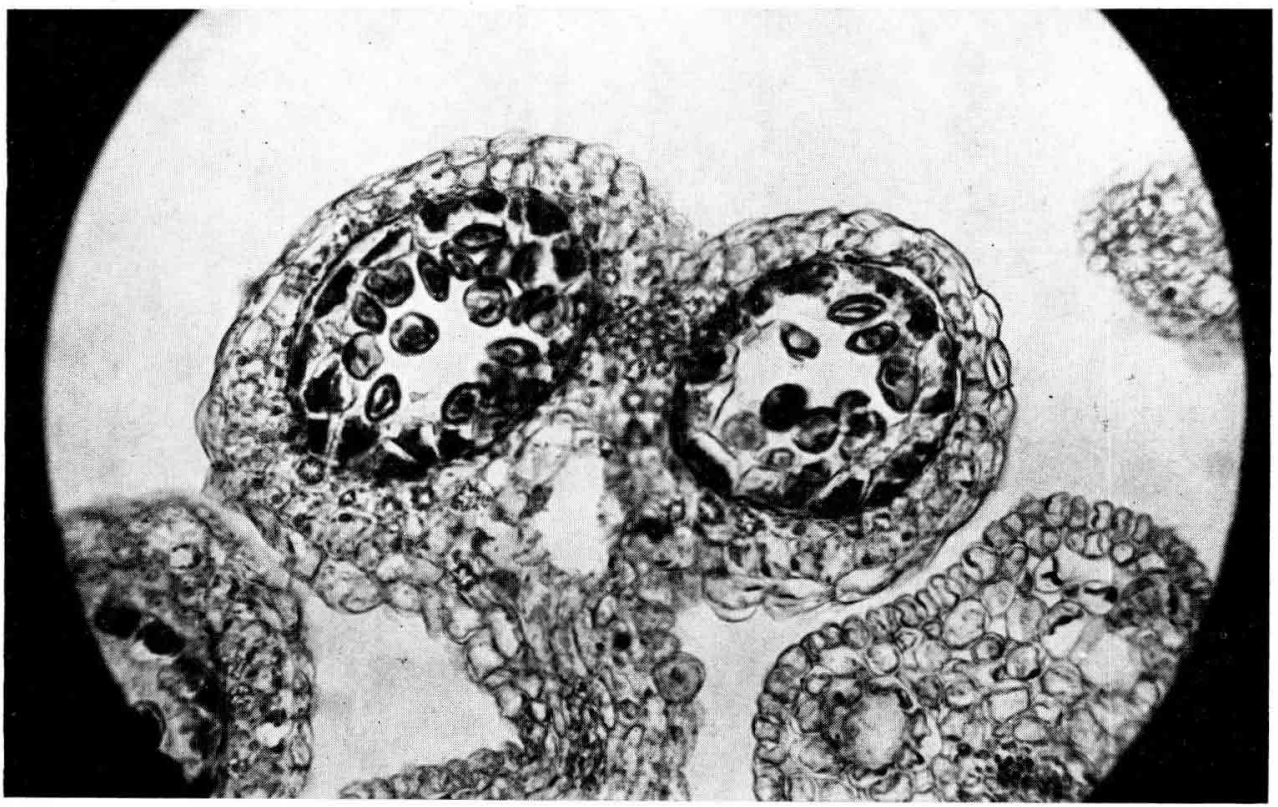

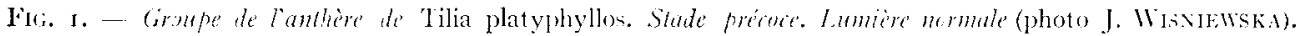

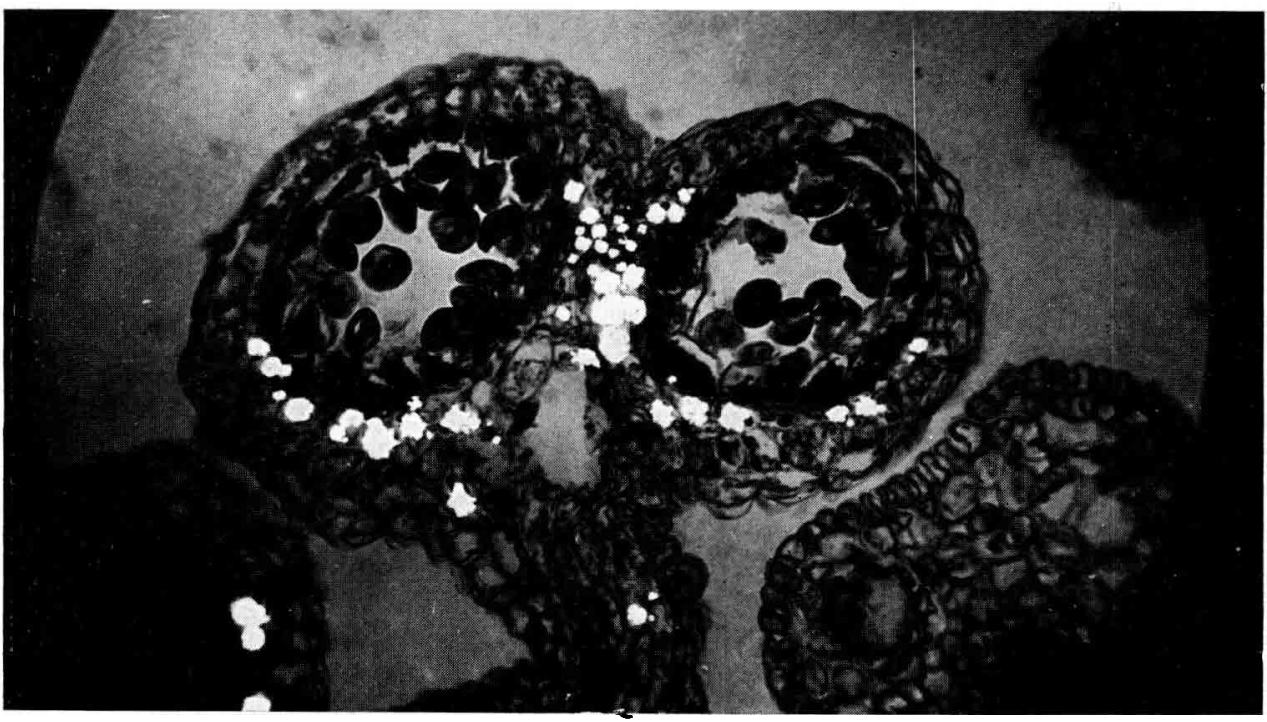

Fug. 2. ... Mene objet que figure I. Lumière polurisée (photo J. Wisciewsisa). 




FIr. 3. Coute de l'anthère de Tilia cordata. Stade tardit. Conche fibreuse bien dózeloppée.

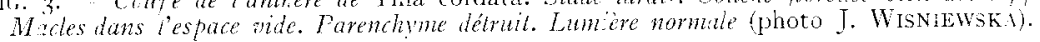

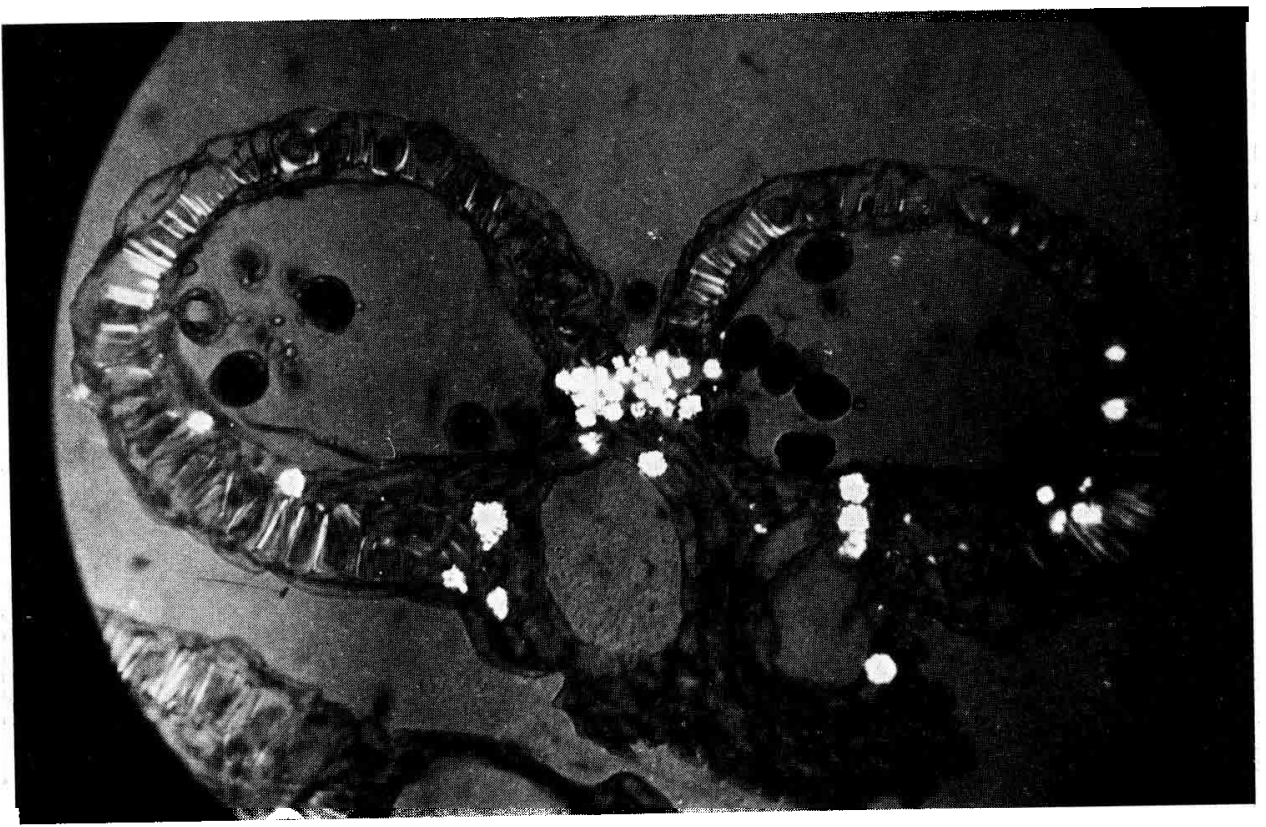

FIG. 4. - Même objet que figure 3. L.es épaississements des parois fibreuses sont bien visibles. Lumière polarisée (photo J. WISNIESWKA). 


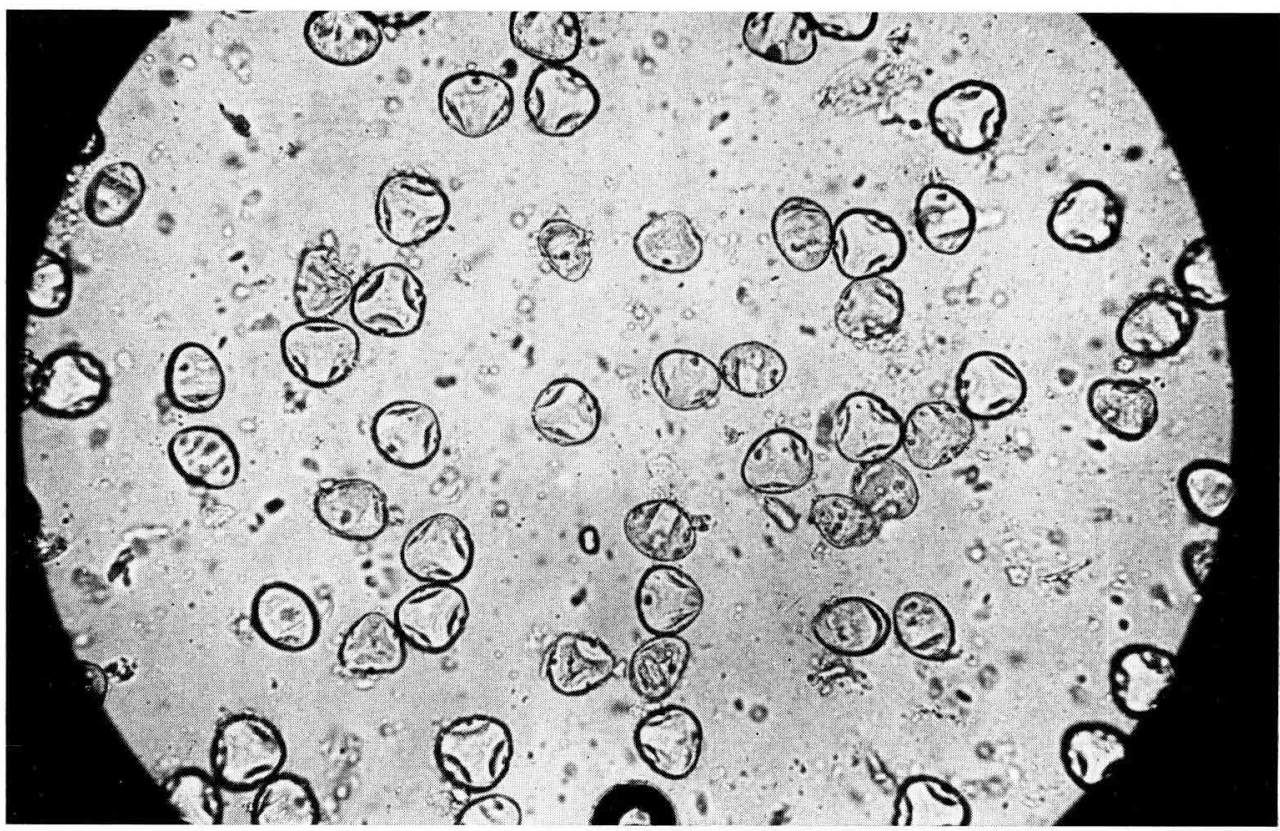

FiG. 5. - Pelote de pollen. Lumière normale (photo J. Wisniewsika).

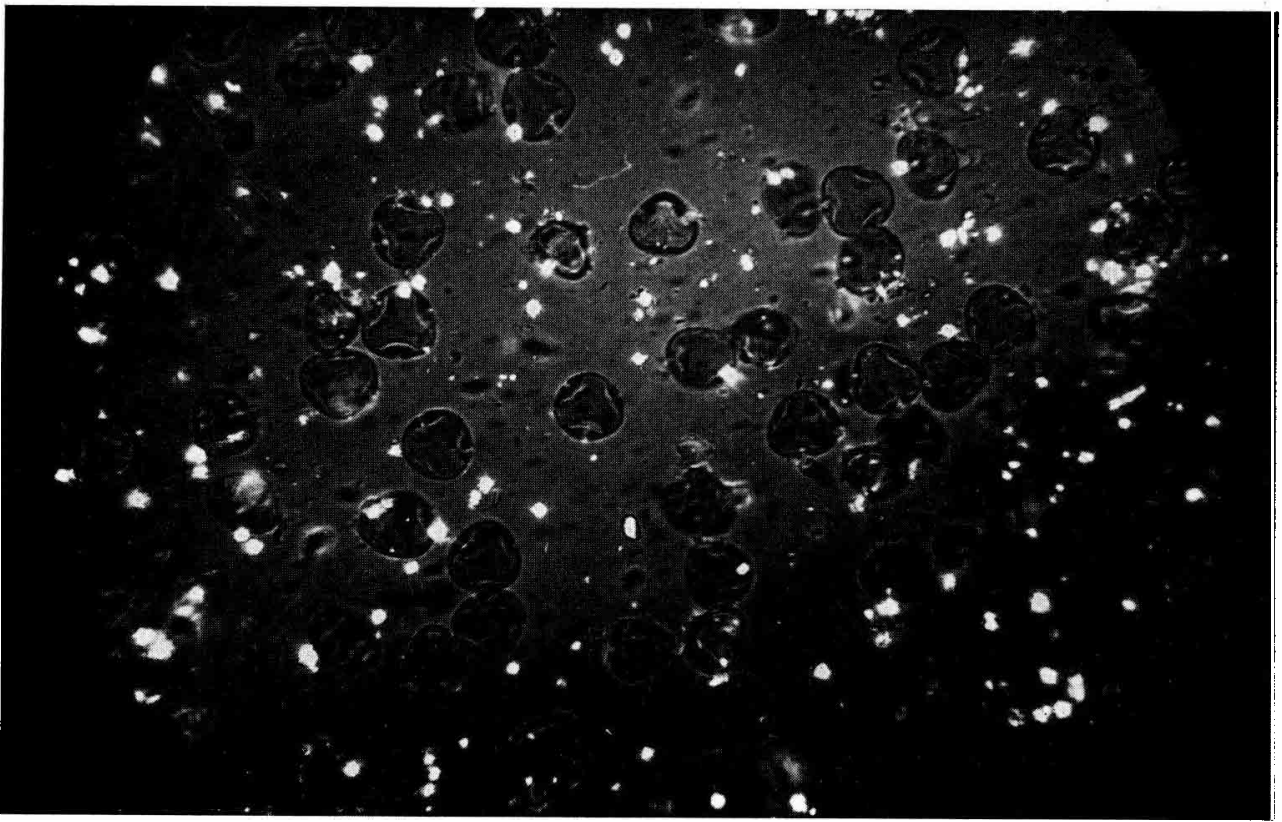

FIG. 6. - Pelote de pollen. Lumière polarisée (photo J. WISNiEswka). 


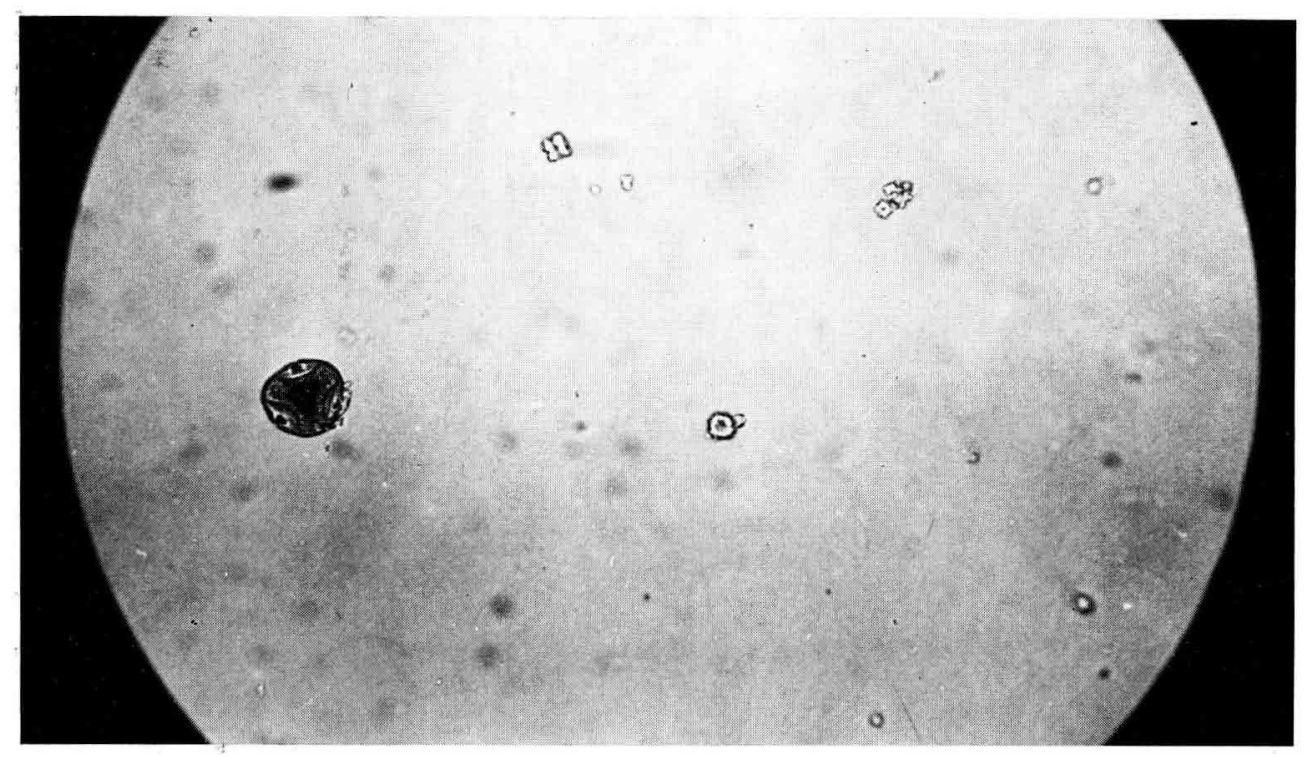

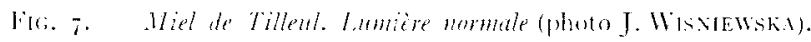

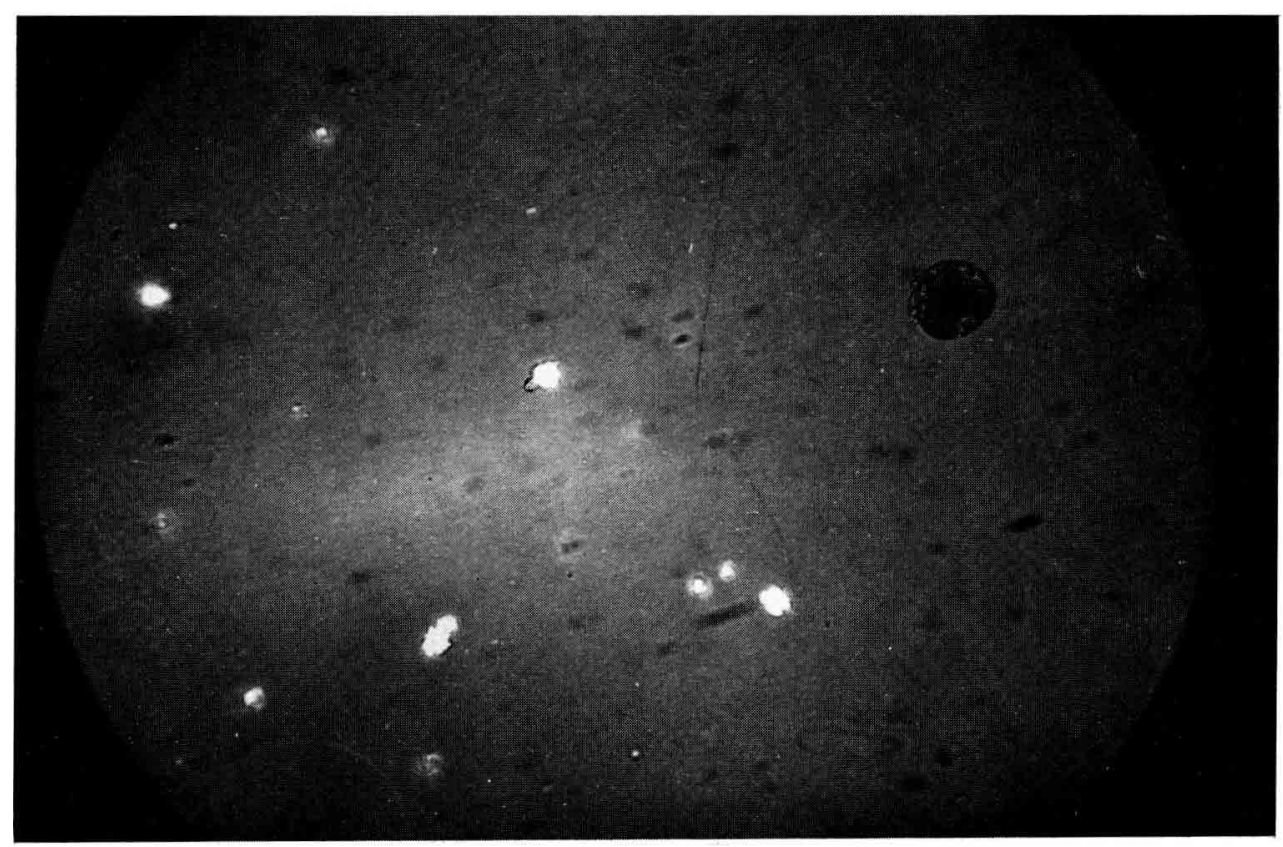

I'Ic. 8. Wiel de Tillend. Lumière polarisee (photo J. Wisviewski). 
Le pollen de Sparmania (f. 'Tiliaceae), en dépit de l'abondance des macles dans la paroi des anthères, n'en contient aucune. Il semble que les macles restent fixées dans les cellules et ne viennent pas en contact avec le pollen.

Mes recherches sur le pollen des principales plantes mellifères, ainsi que celles de Warakomska ( I962) et de LECEWICZ, portant sur les pelotes de follen d'environ I70 plantes mellifères et sur environ 200 échantillons de miel ne provenant pas de Tilleul ont démontré l'absence de macles d'oxalate de calcium.

Il en résulte que les macles d'oxalate de calcium se trouvent exclusivement dans les miels de Tilleul et que, par consáquent, elles peuvent être utilisées, tout comme le pollen, comme élément de diagnostic des miels de cette plante.

Rę̧u pour publication en juin 1963.

\section{SUMMARY}

ON THE ORIGIN OF MACLKS OF CALCICM OXALATE IN TILIA HONEY

The writer had established :

I) that in Tilia macles of calcium oxalate were present in the parenchyma tissue lying between the pollen-sacs ;

2) that these macles became mixed with the pollen as the anthers opened. They could thus be used, like the pollen, for the diagnosis of Tilia honeys.

\section{RÉFÉRENCES BIBI,IOGRAPHIQUES}

DEmavowic\% \%, ig6. Estimation des miels de Tilleul par la méthode des coefficients polliniques Pssczeln. Yess. Vauk., 6 (1), 25-45.

Kuster F.., I956. Die Plimienselle. Jena.

Maurizio A., 1956. P'llenamalyt ische Bechachtungen 13-16. Berichl. d. Schroeiz. Botan. Gesellsch., 66, I 18 - I32.

Gorski F., 1959. Phwsiologie des plantes. P. W. N., Cracovic.

Pelmon C., sgioo. L'examen microcopique du sédiment des miels dans la République populaire Roumaine (dactyl.).

Strasblrger E. et al., igho. Rotanique. Pwril, Varsovie.

Warakouska Z., ig62. Recherches sur lı récolte du pollen par Apis mellifica $L$. dans quelques régions agricoles de la Pologne. Ann. U. M.C.S., 17 (in press I a blin). 